\title{
ON DUAL BRUNN-MINKOWSKI INEQUALITIES
}

\author{
ZHAO CHANG-JIAN, JOSIP PEČARIĆ AND LENG GANG-SONG
}

Abstract. The main purpose of this paper is first to improve two classical dual Brunn-Minkowski inequalities, then we generalize another dual Brunn-Minkowski inequality from generic volume to Quermassintegral.

Mathematics subject classification (2000): 52A40.

Key words and phrases: Dual Brunn-Minkowski inequality, The radial Minkowski linear combination, The Blaschke linear combination..

\section{REFERENCES}

[1] K. BALL, Volume of sections of cubes and related problems, Israel Seminar (G.A.F.A.) 1988, Lecture Notes in Math. Vol. 1376, Springer-Verlag, Berlin and New York, 1989, 251-260.

[2] K. BALL, Shadows of convex bodies Trans. Amer. Math. Soc., Vol. 327, (1991), 891-901.

[3] K. BALL, Volume ratios and a reverse isoperimetric inequality, J. London Math. Soc., Vol. 44, (1991), 351-359.

[4] J. Bourgain, J.Lindenstrauss, Projection bodies, Israel Seminar (G.A.F.A) 1986-1987, Lecture Notes in Math. Vol. 1317, Springer-Verlag, Berlin and New York, 1988, 250-270.

[5] R. J. GARDNER, A positive answer to be Busemann-Petty proplem in three dimensions, Ann. Math., Vol. 140, (1994), 435-477.

[6] R. J. Gardner, Geometric Tomography, Cambridge: Cambridge University Press, 1995.

[7] R. SCHNEIDER, Convex bodies: The Brunn-Minkowski Theory, Cambridge: Cambridge University Press, 1993.

[8] E. LutwaK, Centroid bodies and dual mixed volumes, Proc. London Math. Soc., Vol. 60, (1990), 365-391.

[9] E. LUTWAK, Intersection bodies and dual mixed volumes, Adv. Math., Vol. 71, (1988), $232-261$.

[10] E. LuTWAK, Mixed projection inequalities, Trans. Amer. Math. Soc, Vol. 287, (1985), 92-106.

[11] E. LuTwaK, Dual mixed volumes, Pacific J.Math., Vol. 58, (1975), 531-538.

[12] Leng Gangsong, Zhao ChangJian and He Binwu et al, Inequalities for Polars of Mixed Projection Bodies, Science in China A, 2004, to appear.

[13] Zhao Changuan, On Inverse of Disperse and Continuous Pachpatte's Inequalities, Acta Math. Sin., Vol. 46, (2003), 1248-1254. 